\title{
Amnesia or retrieval deficit? Implications of a molecular approach to the question of reconsolidation
}

\author{
Courtney A. Miller and J. David Sweatt ${ }^{1}$ \\ Department of Neurobiology, University of Alabama at Birmingham, Birmingham, Alabama 35294, USA
}

\begin{abstract}
Post-retrieval interference with a memory has uncovered a phenomenon known to the field as reconsolidation. In this article, we will review the specific molecular mechanisms that have been implicated in reconsolidation. As a result of numerous studies over the past five years, it can now be said with a fair amount of certainty that reconsolidation is not a recapitulation of the mechanisms underlying consolidation, despite what the term "reconsolidation" may suggest. Therefore, in addition to reviewing the known mechanisms of reconsolidation, we will propose that two experimental approaches involving the targeting of specific molecular mechanisms, and the study of these mechanisms during retrieval, may serve useful to the field as it is now able to advance beyond comparisons between consolidation and reconsolidation.
\end{abstract}

First put forth in 1900, the consolidation hypothesis states that new memories are initially unstable and susceptible to both facilitation and impairment, but subsequently strengthen over a discrete period of time (Müller and Pilzecker 1900; McGaugh 1966). Following intense research over the last several decades, this hypothesis has come to incorporate an underlying molecular mechanism for memory consolidation. The requirement of both transcription and translation has become a widely accepted and critical feature of both memory consolidation and formation of its analog in vitro, long-lasting synaptic plasticity.

Over the past century, a wealth of evidence has accrued in support of the consolidation hypothesis. However, Misanin et al. (1968) published data that suggested perhaps there are additional challenges to a memory's stability at the time of retrieval. By following a passive avoidance retention test with electroconvulsive shock (ECS), amnesia for the task was evident in a later retrieval test. The same group later extended their findings to include post-retrieval interference of memory for a complex maze task (Lewis et al. 1972). Judge and Quartermain (1982) were the first to demonstrate that post-retrieval maintenance of memory for an avoidance task is dependent on protein synthesis. The reverse effect, post-retrieval enhancement of a memory, was also reported; Devietti et al. (1977) showed that electrical stimulation of the mesencephalic reticular formation (MRF) improved fear memory when applied after a retrieval test. Taken together, this evidence suggested that post-retrieval modulation of a consolidated memory was a possibility. This idea that not only are new memories initially unstable, but recently reactivated memories are as well, has ignited a battle in the learning and memory field; a battle that was only confounded by the mechanistic connotations of a label first used in 1973, "re-consolidation."

Many have argued, and rightfully so according to the available data, that the term "reconsolidation" is misleading because it implies that the neurobiological processes contributing to retrieval-dependent modulation of a memory recapitulate those that underlie initial memory consolidation. In this article, we will explore the relative paucity of knowledge regarding the spe-

'Corresponding author.

E-mail dsweatt@nrc.uab.edu; fax (205) 975-5097.

Article is online at http://www.learnmem.org/cgi/doi/10.1101//m.304606. cific molecular mechanisms that contribute to reconsolidation and briefly review why it can now be said with a fair amount of certainty that "reconsolidation" is, at most, only a partial recapitulation of consolidation mechanisms (Taubenfeld et al. 2001; Lee et al. 2004; Alberini 2005). But more importantly, we will highlight what we believe to be two currently under-utilized experimental approaches to the question of reconsolidation. The first approach is post-retrieval, targeted disruption or enhancement of specific molecular mechanisms, rather than the use of general protein synthesis inhibitors. The second approach involves studying the molecular mechanisms of retrieval and reconsolidation, rather than perseverating on comparisons between consolidation and reconsolidation. Our description of these approaches will be accompanied by arguments for how they may begin answering a question currently at the heart of the reconsolidation debate.

\section{Reconsolidation revival}

Research pertaining to reconsolidation was revived by the work of Nader et al. (2000), which took a targeted, intra-amygdalar approach to the experiments performed over $30 \mathrm{yr}$ earlier by Misanin et al. (1968). Using what would remain the amnestic agent of choice in the coming years, Nader and colleagues reported that long-lasting memory deficits are produced by intraamygdalar infusions of a protein synthesis inhibitor following retrieval of an auditory fear conditioning memory. Since this publication, a virtual mountain of data has resulted from studies investigating post-retrieval protein synthesis inhibition in a number of brain regions using a variety of behavioral paradigms. Unfortunately, the results of these publications are difficult to reconcile with one another. One particularly troubling disparity comes from reports that the amnestic effect produced by this type of interference is either long-lasting (Nader et al. 2000; Boccia et al. 2005) or, alternatively, subject to spontaneous recovery (Lattal and Abel 2004; Power et al. 2006). This disparity has created two camps in which reconsolidation interference represents either an erasure of the memory trace (long-lasting amnesia) or a retrieval deficit (spontaneous recovery of the memory). However, before we can begin to address any disparities in the field, we must first understand the mechanisms at play when a memory is labile. 


\section{Mechanisms of reconsolidation}

It has now been clearly established that a memory can be interfered with, at least temporarily, by application of either transcriptional or translational inhibitors after retrieval (Lee et al. 2004; Alberini 2005). This section will explore which specific molecular mechanisms these transcriptional and translational inhibitors are likely impinging upon. Figure 1 contains a schematic of the biochemical signaling pathways implicated in reconsolidation thus far.

\section{Receptors}

Initial reports that have looked beyond the necessity of protein synthesis examined the role of various receptors in restabilizing retrieved memories. NMDA receptors have been implicated in the reconsolidation of memories in both vertebrates and invertebrates (Summers et al. 1997; Pedreira et al. 2002) for tasks as varied as odor discrimination and novel object recognition (Torras-Garcia et al. 2005; Akirav and Maroun 2006). Reconsolidation of memory for radial arm maze, inhibitory avoidance, auditory fear conditioning, and sucrose seeking behavior has been shown to also depend upon activation of $\beta$-adrenergic receptors (Przybyslawski et al. 1999; Debiec and LeDoux 2004; Diergaarde et al. 2006). Figure 1 depicts how activation of these receptors can initialize signaling in various pathways, leading to alterations in gene transcription.

\section{Protein kinases}

In addition to the MRF stimulation-induced enhancement of reconsolidation described earlier (Devietti et al. 1977), later studies have demonstrated a similar enhancement of memory using glucose and fructose (Horne et al. 1997; Rodriguez et al. 1999). The demonstration of memory enhancement following retrieval is important because the manipulation provides the field with positive results to interpret. But more importantly, post-retrieval memory enhancement supports the notion that lability after retrieval is a mechanism meant for updating and adjusting the value assigned to memories over time by integrating new information into the memory trace. A recent publication has extended our knowledge of reconsolidation through enhancement by manipulating cAMP-dependent protein kinase A (PKA) activ-

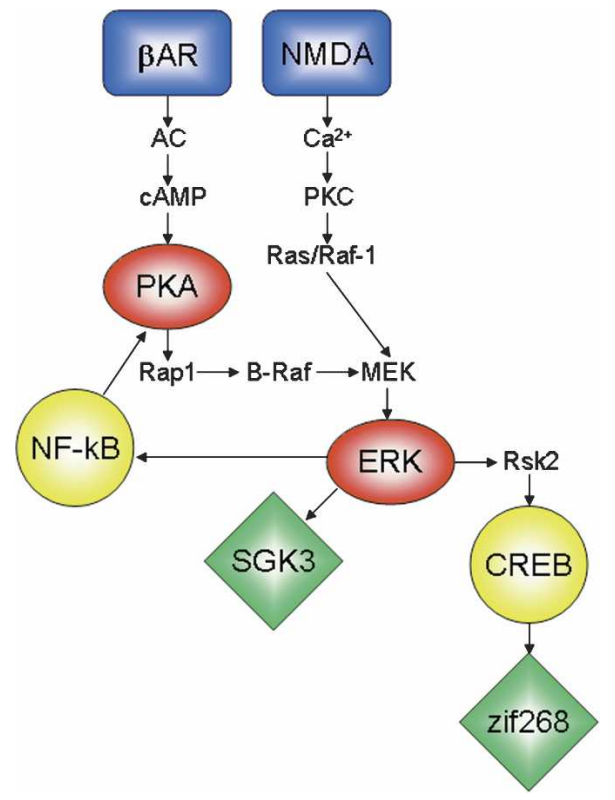

Figure 1. Schematic of the biochemical signaling pathways implicated in reconsolidation. ity following retrieval. Through adenylyl cyclase and cAMP, PKA signaling can be initiated by $\beta$-adrenergic receptor activation (Fig. 1). Tronson et al. (2006) report that post-retrieval intrabasolateral amygdala (BLA) infusions of a specific PKA activator (6-BNZ-CAMP) enhance auditory fear memory at a later retention test. They also demonstrate the reverse effect produced by a PKA inhibitor (Rp-cAMPS) - impairment of memory reconsolidation (Tronson et al. 2006). This latter finding is in agreement with a study published in 2003 that reported intra-amygdala infusions of Rp-cAMPS delivered after a retention test disrupted conditioned taste aversion memory (Koh and Bernstein 2003).

ERK is a member of the mitogen-acitvated protein kinase (MAPK) family that can be indirectly activated by a number of kinases, including PKA. ERK has been implicated in both synaptic plasticity (English and Sweatt 1996; Martin et al. 1997) and the consolidation of numerous learning paradigms (Atkins et al. 1998; Berman et al. 1998; Blum et al. 1999; Hebert and Dash 2002; Kelly et al. 2003). More recently, studies have established that activation of ERK is not only necessary for memory consolidation, but reconsolidation as well. Thus, this kinase is now known to be important for the reconsolidation of traditional memory tasks like object recognition and auditory fear conditioning (Kelly et al. 2003; Duvarci et al. 2005; Cestari et al. 2006).

Researchers in the field recognize that drug addiction is one disorder with a memory component that might greatly benefit from an understanding of how to effectively interfere with memory. The memories for environmental stimuli that have become associated with a drug of abuse, such as cocaine, are highly resistant to extinction and contribute to the high rate of relapse among addicts. Recently two groups have demonstrated that interfering with ERK phosphorylation following retrieval of a memory associating a drug of abuse (cocaine and morphine) with environmental contextual cues results in a long-lasting impairment of that memory (Miller and Marshall 2005; Valjent et al. 2006). These findings suggest that even very strong memories, like those associated with a drug of abuse, are susceptible to disruption. This offers the hope that targeting these memories at the time of retrieval may indeed be a therapeutic option for the treatment of addiction as well as other disorders associated with aberrant memories, such as post-traumatic stress disorder (PTSD) and phobia.

\section{Transcription factors}

An indirect target of ERK is the transcription factor cAMPresponsive element-binding protein (CREB). Using transgenic mice with an inducible and reversible repressor of CREB, Kida et al. (2002) investigated the dependence of memory reconsolidation on CRE-mediated transcription. Following training for contextual fear conditioning, animals were given either vehicle or tamoxifen (TAM) in order to activate an inducible CREBrepressor fusion protein prior to a retrieval test. When tested again $24 \mathrm{~h}$ later, TAM-injected animals displayed significantly less freezing than their vehicle counterparts. This effect was dependent upon re-exposure to the context at the time of TAM treatment; therefore, the necessity of CREB function is necessary for and specific to the stabilization of retrieved memories (Kida et al. 2002)

The role of the transcription factor nuclear factor $\kappa \mathrm{B}(\mathrm{NF}-\kappa \mathrm{B})$ is well characterized in the immune system; however, an understanding of its function in the central nervous system (CNS) is still developing. Accordingly, the transcriptional CNS targets of this factor have yet to be determined. It is, however, already clear that NF- $\mathrm{kB}$ is activated following excitatory synaptic transmission and plays a role in neuronal plasticity and memory consolidation (Merlo et al. 2002; Freudenthal et al. 2005; Mattson 2005). Merlo et al. (2005) have extended the known roles of NF-кB to 
include reconsolidation. The authors found that NF- $\mathrm{B}$ is rapidly induced by exposure to the training context in a crab Chasmagnathus model of associative learning and that inhibition of this transcription factor prevents reconsolidation of the memory. Importantly, the authors demonstrated that the blockade of reconsolidation through NF- $\kappa$ B inhibition was dependent on reexposure to the training context, as the memory was unaffected by NF- $\kappa$ B inhibition when paired with a different context (Merlo et al. 2005). Because of NF-кB's complex signaling, having the capability to act as both a signaling molecule and initiator of transcription, further investigation into NF-кB's exact role in retrieval and reconsolidation processes is likely to produce interesting findings. Perhaps NF- $\mathrm{B}$ serves as a signaling molecule when activated at the synapse to subserve the rapid needs of retrieval, while acting as a transcription factor for the restabilization of a memory once it has been retrieved.

\section{Immediate early genes}

Immediate early genes (IEGs) encode a variety of proteins, including regulatory transcription factors and signal transduction proteins (Lanahan and Worley 1998). The regulatory transcription factors are then capable of affecting transcription of lateresponse genes by interacting with their promoter regulatory regions. In this way, induction of IEGs can affect long-term changes in a cell. Activation of the IEG serum- and gluccocorticoid-induced kinase 3 (SGK3) occurs during both consolidation and reactivation of a contextual fear conditioning memory (von Hertzen and Giese 2005). Interestingly, SGK3 activation is specific to the association between the context and shock, making it an excellent marker of associative memory formation. The authors suggest that, because SGK3 is specific to associative memory formation, the recapitulation of this IEG at the time of reconsolidation lends support to the notion that the amnesia present following post-retrieval inhibitor treatments reported in other studies must reflect more than a retrieval deficit (von Hertzen and Giese 2005).

There is some disagreement about the role of another IEG, zif268 (approved gene symbol Egr1), in consolidation (Jones et al. 2001; Lee et al. 2004), but its activation is clearly necessary for the reconsolidation of memory for object recognition, contextual fear conditioning, and cocaine-paired cues (Bozon et al. 2003; Lee et al. 2004, 2005). If zif268 proves to be activated by, but not necessary for consolidation, then this IEG will be the first molecular mechanism to be identified that is specific to reconsolidation.

While relatively few studies have been published to date investigating the specific molecular mechanisms governing the reconsolidation of a retrieved memory, Figure 1 demonstrates that these studies have managed to characterize processes stretching all of the way from the cell surface (receptor activation), through the cytoplasm (protein kinases) and into the nucleus (transcription factors and IEG transcription). We predict that more focused attention identifying the molecular mechanisms at play when a memory is retrieved will shed further light on this enigmatic process.

\section{Experimental approach \#1: Targeted disruption of specific molecules}

\section{Specific molecules and lasting amnesia}

In reviewing the literature for the specific molecular mechanisms that have been targeted in reconsolidation, we noticed an interesting consistency in the reported findings. All studies that targeted a specific molecule, as opposed to general inhibition of translation, and looked at post-retrieval test time points longer than $24 \mathrm{~h}$ reported long-lasting memory deficits. These studies used both systemic and brain region-specific disruption of three different molecules using both aversive and appetitive tasks (see Table 1 for more specific details of these studies).

It is quite possible that once more investigators target specific molecules in their reconsolidation studies, findings of spontaneous recovery will be reported. However, this observation also suggests a more intriguing possibility-that there is something unique about the function of protein synthesis inhibitors during memory stabilization that is confounding the results.

The discrepancy in length of amnesia reported using protein synthesis inhibitors could be due to a differential sensitivity of the molecules involved in the expression and maintenance of memory if the molecules have different protein half-lives. If this is the case, then the magnitude and duration of inhibition created by the protein synthesis inhibitors becomes very important. While still purely speculative, this model would require that there be entirely different molecules or different concentrations of the same molecules responsible for the expression and the maintenance of a memory. In this model, lower and/or shorter acting concentrations of protein synthesis inhibitors would block production of expression molecules without affecting the maintenance molecules. This would manifest as a memory deficit $24 \mathrm{~h}$ after post-retrieval interference of the memory with protein synthesis inhibitors, but a return at later time points as the maintenance molecules ramp up and support the expression molecules. Greater and/or longer-lasting concentrations of protein synthesis inhibitors would affect both the expression and maintenance molecules-resulting in long-term erasure of the memory trace. Indeed, by using ${ }^{35} \mathrm{~S}$-methionine, Milekic et al. (2006) were able to investigate the magnitude and duration of protein synthesis inhibition produced by a single subcutaneous injection of the protein synthesis inhibitor, cyclohexamide. A

Table 1. Mechanisms tested for long-term post-retrieval memory interference

\begin{tabular}{lccccc}
\hline Behavioral Task & post-retrieval test & $\begin{array}{c}\text { Molecular } \\
\text { target }\end{array}$ & $\begin{array}{c}\text { Route of } \\
\text { administration }\end{array}$ & $\begin{array}{c}\text { Spontaneous } \\
\text { recovery? }\end{array}$ & Reference \\
\hline $\begin{array}{l}\text { Novel object } \\
\text { recognition, mice }\end{array}$ & $5 \mathrm{~d}$ & zif268 & zif268 null mutation & No & No \\
$\begin{array}{c}\text { Contextual fear } \\
\text { conditioning, mice }\end{array}$ & $7 \mathrm{~d}$ & zif268 & Intra-hippocampus & Lee et al. (2004) \\
$\begin{array}{c}\text { Cocaine place } \\
\text { preference, rat }\end{array}$ & $14 \mathrm{~d}$ & ERK & Intra-AcbC & No & Miller and Marshall (2005) \\
$\begin{array}{c}\text { Cocaine place } \\
\text { preference, mice }\end{array}$ & $14 \mathrm{~d}$ & ERK & Systemic & No & Valjent et al. (2006) \\
$\begin{array}{c}\text { Morphine place } \\
\text { preference, mice }\end{array}$ & $14 \mathrm{~d}$ & ERK & Systemic & No & Valjent et al. (2006) \\
$\begin{array}{c}\text { cuditory fear } \\
\text { condioning, rat }\end{array}$ & $7 \mathrm{~d}$ & PKA & Intra-BLA & No & Tronson et al. (2006) \\
\hline
\end{tabular}


single injection of cyclohexamide produced a 70\% inhibition of protein synthesis $1 \mathrm{~h}$ later, but this level had dropped to only $23 \%$ inhibition $6 \mathrm{~h}$ post-injection. However, when two injections were given, separated by $5 \mathrm{~h}$, a 71\% level of inhibition was maintained for all $6 \mathrm{~h}$. Consistent with this idea, animals that received a single injection post-retrieval in a morphine-conditioned place preference paradigm demonstrated a memory deficit at $24 \mathrm{~h}$, but a recovery of the memory at the 1-wk test. However, when the two cyclohexamide injection paradigm was used, the memory deficit showed no sign of recovery, persisting for at least $2 \mathrm{wk}$ (Milekic et al. 2006).

Unfortunately, Milekic and colleagues' approach of measuring levels of protein synthesis inhibition produced by drugs like cyclohexamide and anisomycin is not currently common practice in the field. Therefore, we are unable to look for correlations between the degree of protein synthesis inhibition and the duration of the amnesia reported in different studies. However, considering the various drug doses, routes of administration, and protein synthesis inhibitors used by different investigators, variability in the degree of inhibition from study to study seems likely to exist. This may explain, at least in part, the discrepancy in amnesia duration currently being reported. Perhaps more uniform and reproducible findings would result from targeting specific molecules and demonstrating the level of that molecule's inhibition achieved during the memory's window of instability.

\section{Experimental approach \#2: Studying retrieval mechanisms with reconsolidation}

\section{Reconsolidation is not consolidation repeated}

A review of the molecular mechanisms of reconsolidation outlined earlier cannot help but remind the reader of known consolidation mechanisms. Consolidation requires an entire cascade of intracellular events, including activation of NMDA receptors, an influx of calcium, activation of signaling pathways, including the kinases PKA and ERK, and gene transcription. And, more recently, several labs have discovered an additional, necessary level in the cascade underlying consolidation-epigenetic modulation. Specifically, alterations in chromatin structure and changes in DNA methylation have been associated with consolidation and the underlying synaptic plasticity (Guan et al. 2002; Alarcon et al. 2004; Korzus et al. 2004; Levenson et al. 2004, 2006; Wood et al. 2005). In light of this new facet of memory storage, it will be interesting to see if consolidation and reconsolidation recruit similar or distinct epigenetic mechanisms. If epigenetic mechanisms are active following memory retrieval, this would suggest a relatively substantial handling of the memory trace as it is restabilized.

Despite the mechanistic similarities, reconsolidation is not a recapitulation of consolidation. Table 2 reviews the known reconsolidation mechanisms and compares these with consolidation mechanisms. While the table is by no means intended to be an exhaustive review of consolidation, it does include all mechanisms important for reconsolidation that have been identified to date. Despite the similarities in molecules for the two memory processes, three molecules have been clearly demonstrated as being necessary for consolidation, but not reconsolidation-brain-derived neuMerlo et al. (2005). rotrophic factor (BDNF), CCAAT enhancer-binding protein $(\mathrm{C} /$ $\mathrm{EBP} \beta$ ), and NGI-B (Taubenfeld et al. 2002; Lee et al. 2004; von Hertzen and Giese 2005). And, as mentioned earlier, zif268 appears to be specific to reconsolidation. Lee et al. (2004) report that zif268 is activated during conditioning but is not a required substrate for the formation of associative memory. For these reasons, we know that reconsolidation is not simply a recapitulation of consolidation on the cellular level (for an in depth review of these differences, see Alberini 2005). Rather, the two memory processes have different temporal profiles (post-training vs. postretrieval) and their own molecular signatures.

\section{The impact of retrieval}

Because the field has now clearly established that reconsolidation is a process distinct from consolidation on the cellular level, continued comparisons between these two memory processes may no longer be the ideal experimental approach. Rather, in order to continue investigating reconsolidation, we propose that understanding retrieval, the process that is predicted by the reconsolidation hypothesis to render a memory temporarily unstable, is necessary. We use the term "retrieval" in its most traditional sense to refer to the memory process in which a conditioned behavioral response (CR) is elicited by presentation of a conditioned stimulus (CS) in the absence of the unconditioned stimulus (US). Unfortunately, the molecular mechanisms of memory retrieval are virtually unexplored experimentally, creating a serious challenge for conceptualizing its molecular basis. In the midst of the excitement, the learning and memory field seems to have leapt over the top of the retrieval memory process in order to get from consolidation to reconsolidation. Because of this, retrieval has been largely relegated to the role of behavioral readout for interpreting consolidation and reconsolidation manipulations. In light of how little we know about retrieval, it might be useful to review what we know from first principles and integrate them with the few available experimental data directly relevant to molecular mechanisms of retrieval.

One important point to make is that we should consider the strong impact of retrieval on the molecular basis of storage per se. As Dudai (2002) has stated in Memory from A to Z: "Retrieval is not merely a passive readout of information, it is also an experience; therefore, once retrieved the engram is unlikely to remain exactly the same." This is a valid and compelling point not only from a cognitive perspective, but also from a molecular perspective. The

Table 2. Mechanisms of consolidation versus reconsolidation

\begin{tabular}{lcccc}
\hline Mechanism & Consolidation? & Reference $^{\mathbf{a}}$ & Reconsolidation? $^{\text {Reference }^{\mathbf{a}}}$ \\
\hline NMDA & Yes & $1-2$ & Yes & $14-17$ \\
$\beta$-AR & Yes & $3-4$ & Yes & $3-4,18-19$ \\
PKA & Yes & 5 & Yes & $20-21$ \\
ERK & Yes & 6 & Yes & $22-25$ \\
NF-KB & Yes & $7-8$ & Yes & 26 \\
CREB & Yes & 9 & Yes & 9 \\
C/EBPB & Yes & 10 & No & 10 \\
zif268 & $?$ & $11-12$ & Yes & 11 \\
SGK3 & Yes & 13 & Yes & 13 \\
NGI-B & Yes & 13 & No & 13 \\
BDNF & Yes & 11 & No & 11 \\
\hline
\end{tabular}

Mechanisms in bold are not common to both consolidation and reconsolidation.

a (1) Steele and Morris (1999); (2) Shimizu et al. (2000); (3) Przybyslawski et al. (1999); (4) Roullet and Sara (1998); (5) Arnsten et al. (2005); (6) Sweatt (2004); (7) Freudenthal et al. (2005); (8) Yeh et al. (2002); (9) Kida et al. (2002); (10) Taubenfeld et al. (2001); (11) Lee et al. (2004); (12) Bozon et al. (2003); (13) von Hertzen and Giese (2005); (14) Summers et al. (1997); (15) Torras-Garcia et al. (2005); (16) Pedreira et al. (2002); (17) Akirav and Maroun (2006); (18) Diergaarde et al. (2006); (19) Debiec and LeDoux (2004); (20) Koh and Bernstein (2003); (21) Tronson et al. (2006); (22) Kelly et al. (2003); (23) Duvarci et al. (2005); (24) Miller and Marshall (2005); (25) Valjent et al. (2006); (26) 
molecular mechanisms of retrieval likely impinge directly upon the molecular basis of storing the engram. We believe that this is an important consideration to incorporate into any model explaining the lability of recalled memories.

Second, we know from molecular studies of retrieval that this seemingly simple process triggers large-magnitude molecular changes in the brain. These molecular changes triggered by retrieval are of a large enough magnitude, and involve a sufficiently high number of neurons, to be able to be directly measured biochemically. For example, protein kinase phosphorylation, transcription factor activation, and altered gene expression have been directly measured in CNS extracts after behaviorally manifested memory retrieval. Specific mechanisms identified as functioning at the time of retrieval include phosphatidylinositol 3 kinase activation, ERK and CREB phosphorylation, and transcription of the IEGs c-fos, JunB, and Arc (Hall et al. 2001; Strekalova et al. 2003; Mizoguchi et al. 2004; Chen et al. 2005; Miller and Marshall 2005; Zhang et al. 2005). Thus, in contrast to what might be our preconceived notion, that is that retrieval in molecular terms is mundane and pedestrian, retrieval itself is a high impact event at the molecular level.

Third, from a biochemical perspective, we are able to draw at least one conclusion about the fundamental chemical nature of the information storage process. As has been described in greater detail in a prior publication from our research group (Roberson and Sweatt 1999; Sweatt 2003), we are able to deduce that a certain, specific type of chemical reaction must be involved in long-term memory maintenance in order to overcome the problem of constant molecular turnover. This reaction has the following general form:

$$
\mathrm{X}+\mathrm{X}^{*} \rightarrow \mathrm{X}^{*}+\mathrm{X}^{*}
$$

In this equation, a mechanism associated with learning initially converts $\mathrm{X}$ from its basal state to an activated form $\left(\mathrm{X}^{*}\right)$. However, once activated, $X^{*}$ is itself capable of catalyzing conversion of another molecule of $X$ to $X^{*}$ as part of a positive feedback loop. This formula describes a self-perpetuating chemical reaction, which allows a molecule to pass along its acquired characteristics to a successor molecule. This is the only type of biochemical reaction capable of sustaining itself in the face of protein turnover, making it the essential biochemical reaction of the engram. Known examples of this type of reaction include epigenetic mechanisms like chromatin modification and DNA methylation, prion autoconversion, and transcription factor autoregulation (Saitoh and Schwartz 1985; Lai et al. 1986; Miller and Kennedy 1986; Prusiner 1998; Turner 2002; Si et al. 2003a,b; Levenson and Sweatt 2006). Therefore, when we discuss a retrieval mechanism impinging upon a biochemical storage mechanism, we know that the retrieval mechanism must perturb at least one chemical reaction of this form. It is quite possible that this impingement on the chemical reaction capable of sustaining itself in the face of protein turnover is what renders a memory unstable and in need of reconsolidation following retrieval.

Once one has considered the impact of retrieval on the brain, the importance of the right experimental design for investigating this memory process becomes clear. It is obvious that one cannot study the mechanisms of retrieval without contamination from acquisition and consolidation processes if a manipulation (e.g., gene expression manipulation or lesion) is performed at or before the time of training. Rather, in order to isolate retrieval mechanisms, any manipulation must be performed immediately prior to the retrieval test. A second consideration that must be incorporated into the design involves the timecourse of specific molecules activated by acquisition and consolidation. Prior to manipulation of a molecule at the time of retrieval, the experimenter must first establish either (1) that the molecule was not activated by acquisition of consolidation of the memory at the time of training or (2) that the molecule has returned to basal activation levels by the time a retrieval manipulation and test are performed. If the timecourse of a molecule's training-induced activation is not accounted for, any lasting effect of a manipulation at the time of retrieval could be explained as an effect on consolidation, not a manipulation of retrieval-specific events.

\section{Understanding reconsolidation through retrieval}

Some interpret the disparity in amnesia duration as grounds for challenging the existence of reconsolidation. However, we would like to suggest that these experimental differences may actually provide an excellent opportunity to determine how closely the molecular events associated with memory retrieval and restabilization mirror the behavioral read-out in retention tests. By correlating the sizeable molecular events that occur at the time of retrieval with behavioral output, we may be able to extract some information about how post-retrieval interference of a memory works.

Surprisingly few studies have examined the effect of postretrieval experimental manipulations on recruitment of retrievalassociated signaling molecules during subsequent retention tests. Examining reconsolidation in terms of specific molecular mechanisms, rather than general protein synthesis inhibition, will identify molecular events important to memory retrieval for that task. By doing so, it may be possible to ask if the molecular activation observed following the first retrieval test is altered in conjunction with the behavior. If cellular activation is altered, do these molecular events return in instances of spontaneous recovery of the memory? In other words, do the alterations in molecular activation mirror the alterations in behavior following postretrieval memory interference?

A common experimental approach used to identify a potential molecular candidate important for reconsolidation is to assess molecular events that occur during the initial retrieval test. However, to our knowledge, only two studies have examined how post-retrieval interference with one of these molecules affects not only the behavioral read-out, but the molecule's activation at later retention tests. Both of these studies happened to target ERK in the reconsolidation of memory using a model of contextual cue-elicited drug seeking. In this model, known as conditioned place preference (CPP), rats learn to associate the rewarding effects of a drug of abuse, such as cocaine, with the environmental context in which it is administered and later show a preference for that environment.

In the first study, Miller and Marshall (2005) trained animals in a CPP apparatus to associate one chamber with the rewarding effects of a cocaine injection. Expression of a place preference at a retrieval test administered $48 \mathrm{~h}$ later was accompanied by increased activation of ERK, two downstream transcription factors, CREB and ets-like gene-1 (Elk-1), and the IEG c-fos in a subregion of the nucleus accumbens, the core (AcbC). After identifying ERK as a molecule activated by retrieval of this type of memory, the authors targeted its activation in the AcbC in a second group of animals following a CPP retrieval test. Animals were trained for $\mathrm{CPP}$, tested $48 \mathrm{~h}$ later, and then given intra-AcbC infusions of a vehicle or an inhibitor (U0126) of ERK's upstream activating kinase, MEK.

Two pieces of this study are particularly germane to the current discussion. First, following a subsequent retrieval test 24 $\mathrm{h}$ later, the authors compared activation of the ERK pathway $($ ERK $\rightarrow$ CREB/Elk-1 $\rightarrow$ c-fos) in the MEK inhibitor-treated animals with their vehicle counterparts. Unlike the vehicle-treated animals, those that received U0126 in conjunction with a retrieval test the previous day failed to show a place preference in the

\section{Learning \& Memory}


second test. Interestingly, activation of the ERK pathway following this post-retrieval interference test mirrored the behavior. While vehicle-treated animals showed significant activation of the ERK pathway, this was completely absent in the U0126 animals. Using a second group of animals, Miller and Marshall observed this same phenomenon at a 14-d post-retrieval interference test. By studying both the behavioral and molecular changes present at subsequent retrieval tests, these experiments demonstrate a long-lasting U0126-induced blockade of both the memory, according to the absence of a behavioral read-out of the memory, and activation of the ERK pathway. In this case, the behavioral read-out and molecular changes mirrored each other, an effect one would expect if the memory trace had been permanently altered by post-retrieval interference.

In a similar study, Valjent et al. (2006) also used cocaine CPP to investigate the possibility that ERK inhibition might interrupt memory reconsolidation. Following a retrieval test, animals were given a systemic injection of another MEK inhibitor, SL327, or vehicle. Relative to vehicle-treated and no injection controls, SL327 prevented expression of a place preference when the animals were tested the following day. Importantly, in addition to the behavioral deficit, the Acb ERK phosphorylation induced by the retrieval test in control animals was absent in the SL327treated animals. In addition to ERK, the authors examined phosphorylation of an AMPA glutamate receptor subunit, glutamate receptor-1 (GluR1), at the retention test $24 \mathrm{~h}$ after post-retrieval interference with SL327. GluR1 is phosphorylated in response to cocaine treatment through PKA activation (Snyder et al. 2000). A cocaine CPP retrieval test induces phosphorylation of GluR1 in the Acb and dorsal striatum, an effect that is prevented in animals that had previously received SL327 paired with a retrieval test (Valjent et al. 2006). Again, the behavioral read-out and molecular changes brought about by post-retrieval interference with the memory mirrored one another at the subsequent retrieval test, suggesting a permanent alteration of the memory trace. It is possible that the MEK inhibition in both studies is affecting the subsequent ability of the animal to retrieve the memory trace, rather than altering it. However, this seems unlikely, as it would require that the MEK inhibitor still be functionally active during the subsequent retention tests, which have been performed both $24 \mathrm{~h}$ and $14 \mathrm{~d}$ after drug delivery. We are not aware of a mechanism through which acute inhibition of ERK could interfere with the active process of memory retrieval two weeks later. It seems more likely that the MEK inhibitor would have an effect on the return of a memory to storage at the time of treatment, which was manifested during the retention test two weeks later.

These two studies demonstrate that blocking the behavioral read-out with post-retrieval ERK inhibition coincides with a lack of molecular activation, suggesting a true amnesia for the memory, rather than retrieval-deficit. However, because in both instances the memory deficit was long-lasting, these studies are only able to address half of the issue. It would be very interesting to investigate activation of the ERK pathway, or any other specific reconsolidation mechanism, in a paradigm that has been reported to result in spontaneous recovery of the memory. One would predict that ERK activation would be present at the initial retrieval test, prior to reconsolidation interference, and absent at the $24-\mathrm{h}$ retention when the field agrees that the behavioral readout is absent. However, predicting the activation pattern of ERK at the later test, when spontaneous recovery has been observed, is more difficult. Would ERK inhibition, like protein synthesis inhibition, fail to induce a long-lasting memory deficit? If so, would the ERK activation return with the memory? This would have implications for the lost memory trace versus retrieval deficit debate.

Perhaps return of the memory reflects a ramping up of maintenance molecules that were not sufficiently inhibited after the initial retrieval test, as we suggested in an earlier section. If this were the case, we would predict a return of ERK activation, accompanying the return of the memory's behavioral manifestation. Would this then be interpreted as a retrieval deficit?

It is possible that examination of molecular events throughout the memory's entire neural circuit during retrieval tests may clarify whether the memory trace is absent or the animals are indeed suffering from a retrieval deficit. This might be manifest as cellular activation in upstream brain regions, but not ones closer to behavioral motor output.

Interestingly, in the Valjent and colleagues' study, blockade of reconsolidation was accompanied by a decrease in ERK phosphorylation in more brain regions than the Acb. ERK signaling was also affected in the dorsal striatum and prefrontal cortex of these animals (Valjent et al. 2006). This suggests that interference with ERK activation at the time of memory retrieval affects activation of ERK throughout the circuitry involved in CPP.

The CPP task is relatively complex because of the number of cues that can serve as conditioned stimuli (CS) to the animal. For this reason, retrieval of this type of memory engages a number of brain structures, including the BLA, prefrontal cortex, hippocampus, and Acb (Weiss et al. 2000; Carelli and Ijames 2001; Meyers et al. 2003; Fuchs et al. 2004; Miller and Marshall 2004, 2005). It is likely that these structures cooperate as a neural circuit to form the memory trace by storing different components of the memory. In light of this and the findings of decreased ERK activation in multiple brain structures by Valjent et al. (2006), it would be very interesting to explore whether or not post-retrieval ERK disruption targeting a specific brain region also affects the kinase's activation pattern in other brain regions. This has the potential to provide valuable information about how postretrieval interference affects the memory trace. For example, it is possible that post-retrieval inhibition of ERK within the Acb would erase only the portion of a memory trace that is stored there. Thus, subsequent exposure to the CS would still activate memory via the appropriate signaling pathways in other regions of the circuit storing different portions of the memory. The important question for predicting the behavioral read-out then becomes whether or not the portions of the memory trace that remain are enough for the animal to display the appropriate conditioned response.

If this is how interference with reconsolidation is working on a systems level, what does this mean in terms of the amnesia versus retrieval deficit question? In this model, it would appear that both are accurate, but applicable to different parts of the brain and the portion of the memory trace stored there. The answer really then depends on one's definition of amnesia. Because we cannot ask a rat or mouse if they remember receiving cocaine or being shocked in a specific context, we are forced to rely solely on the behavioral read-out. In this way, if interruption of a portion of a memory trace in one brain region is enough to interfere with the conditioned response, then the interpretation would be amnesia. However, if signaling pathways are still activated in brain regions holding the remaining portions of the memory trace, then on the cellular level, we would be observing a retrieval-deficit.

\section{Molecular insight into reconsolidation}

In this review, we have described the specific molecular mechanisms that have been implicated in the restabilization of a retrieved memory and described two experimental approaches that may serve useful in advancing our understanding of the reconsolidation phenomenon. While it is beyond our capacity to answer the fundamental amnesia versus retrieval deficit question that has vexed the field for $40 \mathrm{yr}$, we hope that the molecular 
perspective reviewed here will lend itself to an eventual understanding of the issue. In this review, we have suggested that researchers consider targeting specific molecular mechanisms following retrieval of a memory. Probing what effect this has on the molecule's activation pattern in subsequent retention tests may help to clarify the amnesia versus retrieval deficit question currently being debated, by providing a second level of analysis to supplement measures of the behavioral read-out. Thus far, in studies of this sort, it appears that, at a minimum, disruption of reconsolidation leads to an actual disruption of the molecular memory trace, and that disruption of recall is insufficient to explain the molecular results.

\section{References}

Akirav, I. and Maroun, M. 2006. Ventromedial prefrontal cortex is obligatory for consolidation and reconsolidation of object recognition memory. Cereb. Cortex http://cercor.oxfordjournals.org/ cgi/content/abstract/bhj114v1.

Alarcon, J.M., Malleret, G., Touzani, K., Vronskaya, S., Ishii, S., Kandel, E.R., and Barco, A. 2004. Chromatin acetylation, memory, and LTP are impaired in $\mathrm{CBP}^{+/-}$mice: A model for the cognitive deficit in Rubinstein-Taybi syndrome and its amelioration. Neuron 42: 947-959.

Alberini, C.M. 2005. Mechanisms of memory stabilization: Are consolidation and reconsolidation similar or distinct processes? Trends Neurosci. 28: 51-56.

Arnsten, A.F., Ramos, B.P., Birnbaum, S.G., and Taylor, J.R. 2005 Protein kinase A as a therapeutic target for memory disorders: Rationale and challenges. Trends Mol. Med. 11: 121-128.

Atkins, C.M., Selcher, J.C., Petraitis, J.J., Trzaskos, J.M., and Sweatt, J.D. 1998. The MAPK cascade is required for mammalian associative learning. Nat. Neurosci. 1: 602-609.

Berman, D.E., Hazvi, S., Rosenblum, K., Seger, R., and Dudai, Y. 1998. Specific and differential activation of mitogen-activated protein kinase cascades by unfamiliar taste in the insular cortex of the behaving rat. J. Neurosci. 18: 10037-10044.

Blum, S., Moore, A.N., Adams, F., and Dash, P.K. 1999. A mitogen-activated protein kinase cascade in the CA1/CA2 subfield of the dorsal hippocampus is essential for long-term spatial memory. $J$. Neurosci. 19: 3535-3544.

Boccia, M.M., Blake, M.G., Acosta, G.B., and Baratti, C.M. 2005 Memory consolidation and reconsolidation of an inhibitory avoidance task in mice: Effects of a new different learning task. Neuroscience 135: 19-29.

Bozon, B., Davis, S., and Laroche, S. 2003. A requirement for the immediate early gene zif268 in reconsolidation of recognition memory after retrieval. Neuron 40: 695-701.

Carelli, R.M. and Ijames, S.G. 2001. Selective activation of accumbens neurons by cocaine-associated stimuli during a water/cocaine multiple schedule. Brain Res. 907: 156-161.

Cestari, V., Costanzi, M., Castellano, C., and Rossi-Arnaud, C. 2006. A role for ERK2 in reconsolidation of fear memories in mice. Neurobiol. Learn. Mem. (in press).

Chen, X., Garelick, M.G., Wang, H., Lil, V., Athos, J., and Storm, D.R. 2005. PI3 kinase signaling is required for retrieval and extinction of contextual memory. Nat. Neurosci. 8: 925-931.

Debiec, J. and LeDoux, J.E. 2004. Disruption of reconsolidation but not consolidation of auditory fear conditioning by noradrenergic blockade in the amygdala. Neuroscience 129: 267-272.

Devietti, T.L., Conger, G.L., and Kirkpatrick, B.R. 1977. Comparison of the enhancement gradients of retention obtained with stimulation of the mesencephalic reticular formation after training or memory reactivation. Physiol. Behav. 19: 549-554.

Diergaarde, L., Schoffelmeer, A.N., and De Vries, T.J. 2006. $\beta$-adrenoceptor mediated inhibition of long-term reward-related memory reconsolidation. Behav. Brain Res. (in press).

Dudai, Y. 2002. Memory from A to Z. Keywords, concepts and beyond. Oxford University Press, Oxford, UK.

Duvarci, S., Nader, K., and LeDoux, J.E. 2005. Activation of extracellular signal-regulated kinase-mitogen-activated protein kinase cascade in the amygdala is required for memory reconsolidation of auditory fear conditioning. Eur. J. Neurosci. 21: 283-289.

English, J.D. and Sweatt, J.D. 1996. Activation of p42 mitogen-activated protein kinase in hippocampal long term potentiation. J. Biol. Chem. 271: 24329-24332.

Freudenthal, R., Boccia, M.M., Acosta, G.B., Blake, M.G., Merlo, E., Baratti, C.M., and Romano, A. 2005. NF- $\mathrm{kB}$ transcription factor is required for inhibitory avoidance long-term memory in mice. Eur. J.
Neurosci. 21: 2845-2852.

Fuchs, R.A., Evans, K.A., Parker, M.C., and See, R.E. 2004. Differential involvement of the core and shell subregions of the nucleus accumbens in conditioned cue-induced reinstatement of cocaine seeking in rats. Psychopharmacology 176: 459-465.

Guan, Z., Giustetto, M., Lomvardas, S., Kim, J.H., Miniaci, M.C., Schwartz, J.H., Thanos, D., and Kandel, E.R. 2002. Integration of long-term-memory-related synaptic plasticity involves bidirectional regulation of gene expression and chromatin structure. Cell 111: 483-493.

Hall, J., Thomas, K.L., and Everitt, B.J. 2001. Fear memory retrieval induces CREB phosphorylation and Fos expression within the amygdala. Eur. J. Neurosci. 13: 1453-1458.

Hebert, A.E. and Dash, P.K. 2002. Extracellular signal-regulated kinase activity in the entorhinal cortex is necessary for long-term spatial memory. Learn. Mem. 9: 156-166.

Horne, C.A., Rodriguez, W.A., Wright, T.P., and Padilla, J.L. 1997. Time-dependent effects of fructose on the modulation of a reactivated memory. Prog. Neuropsychopharmacol. Biol. Psychiatry 21: $649-658$.

Jones, M.W., Errington, M.L., French, P.J., Fine, A., Bliss, T.V., Garel, S., Charnay, P., Bozon, B., Laroche, S., and Davis, S. 2001. A requirement for the immediate early gene zif268 in the expression of late LTP and long-term memories. Nat. Neurosci. 4: 289-296.

Judge, M.E. and Quartermain, D. 1982. Characteristics of retrograde amnesia following reactivation of memory in mice. Physiol. Behav. 28: 585-590.

Kelly, A., Laroche, S., and Davis, S. 2003. Activation of mitogen-activated protein kinase/extracellular signal-regulated kinase in hippocampal circuitry is required for consolidation and reconsolidation of recognition memory. J. Neurosci. 23: 5354-5360.

Kida, S., Josselyn, S.A., de Ortiz, S.P., Kogan, J.H., Chevere, I., Masushige, S., and Silva, A.J. 2002. CREB required for the stability of new and reactivated fear memories. Nat. Neurosci. 5: 348-355.

Koh, M.T. and Bernstein, I.L. 2003. Inhibition of protein kinase A activity during conditioned taste aversion retrieval: Interference with extinction or reconsolidation of a memory? Neuroreport 14: 405-407.

Korzus, E., Rosenfeld, M.G., and Mayford, M. 2004. CBP histone acetyltransferase activity is a critical component of memory consolidation. Neuron 42: 961-972.

Lai, Y., Nairn, A.C., and Greengard, P. 1986. Autophosphorylation reversibly regulates the $\mathrm{Ca}^{2+}$ /calmodulin-dependence of $\mathrm{Ca}^{2+} /$ calmodulin-dependent protein kinase II. Proc. Natl. Acad. Sci. 83: $4253-4257$.

Lanahan, A. and Worley, P. 1998. Immediate-early genes and synaptic function. Neurobiol. Learn. Mem. 70: $37-43$.

Lattal, K.M. and Abel, T. 2004. Behavioral impairments caused by injections of the protein synthesis inhibitor anisomycin after contextual retrieval reverse with time. Proc. Natl. Acad. Sci. 101: 4667-4672.

Lee, J.L., Everitt, B.J., and Thomas, K.L. 2004. Independent cellular processes for hippocampal memory consolidation and reconsolidation. Science 304: 839-843.

Lee, J.L., Di Ciano, P., Thomas, K.L., and Everitt, B.J. 2005. Disrupting reconsolidation of drug memories reduces cocaine-seeking behavior. Neuron 47: 795-801.

Levenson, J.M. and Sweatt, J.D. 2006. Epigenetic mechanisms: A common theme in vertebrate and invertebrate memory formation. Cell. Mol. Life Sci. 63: 1009-1016.

Levenson, J.M., O’Riordan, K.J., Brown, K.D., Trinh, M.A., Molfese, D.L., and Sweatt, J.D. 2004. Regulation of histone acetylation during memory formation in the hippocampus. J. Biol. Chem. 279: 40545-40559.

Levenson, J.M., Roth, T.L., Lubin, F.D., Miller, C.A., Huang, I.C., Desai, P., Malone, L.M., and Sweatt, J.D. 2006. Evidence that DNA (Cytosine-5) methyltransferase regulates synaptic plasticity in the hippocampus. J. Biol. Chem. 281: 15763-15773.

Lewis, D.J., Bregman, N.J., and Mahan Jr., J.J. 1972. Cue-dependent amnesia in rats. J. Comp. Physiol. Psychol. 81: 243-247.

Martin, K.C., Michael, D., Rose, J.C., Barad, M., Casadio, A., Zhu, H., and Kandel, E.R. 1997. MAP kinase translocates into the nucleus of the presynaptic cell and is required for long-term facilitation in Aplysia. Neuron 18: 899-912.

Mattson, M.P. 2005. NF-кB in the survival and plasticity of neurons. Neurochem. Res. 30: 883-893.

McGaugh, J.L. 1966. Time-dependent processes in memory storage. Science 153: 1351-1358.

Merlo, E., Freudenthal, R., and Romano, A. 2002. The ІкB kinase inhibitor sulfasalazine impairs long-term memory in the crab Chasmagnathus. Neuroscience 112: 161-172.

Merlo, E., Freudenthal, R., Maldonado, H., and Romano, A. 2005. Activation of the transcription factor NF- $\mathrm{B}$ by retrieval is required 
for long-term memory reconsolidation. Learn. Mem. 12: 23-29.

Meyers, R.A., Zavala, A.R., and Neisewander, J.L. 2003. Dorsal, but not ventral, hippocampal lesions disrupt cocaine place conditioning. Neuroreport 14: 2127-2131.

Milekic, M.H., Brown, S.D., Castellini, C., and Alberini, C.M. 2006. Persistent disruption of an established morphine conditioned place preference. I. Neurosci. 26: 3010-3020.

Miller, S.G. and Kennedy, M.B. 1986. Regulation of brain type II $\mathrm{Ca}^{2+} /$ calmodulin dependent protein kinase by autophosphorylation: A Ca ${ }^{2+}$-triggered molecular switch. Cell 44: 861-870.

Miller, C.A. and Marshall, J.F. 2004. Altered prelimbic cortex output during cue-elicited drug seeking. J. Neurosci. 24: 6889-6897.

. 2005. Molecular substrates for retrieval and reconsolidation of cocaine-associated contextual memory. Neuron 47: 873-884.

Misanin, J.R., Miller, R.R., and Lewis, D.J. 1968. Retrograde amnesia produced by electroconvulsive shock after reactivation of a consolidated memory trace. Science 160: 554-555.

Mizoguchi, H., Yamada, K., Mizuno, M., Mizuno, T., Nitta, A., Noda, Y., and Nabeshima, T. 2004. Regulations of methamphetamine reward by extracellular signal-regulated kinase 1/2/ets-like gene-1 signaling pathway via the activation of dopamine receptors. Mol. Pharmacol. 65: $1293-1301$.

Müller, G.E. and Pilzecker, A. 1900. Experimentelle Beiträge zur Lehre vom Gedächtnis. Z. Psychol. 1: 1-288.

Nader, K., Schafe, G.E., and Le Doux, J.E. 2000. Fear memories require protein synthesis in the amygdala for reconsolidation after retrieval. Nature 406: 722-726.

Pedreira, M.E., Perez-Cuesta, L.M., and Maldonado, H. 2002. Reactivation and reconsolidation of long-term memory in the crab Chasmagnathus: Protein synthesis requirement and mediation by NMDA-type glutamatergic receptors. J. Neurosci. 22: 8305-8311.

Power, A.E., Berlau, D.J., McGaugh, J.L., and Steward, O. 2006. Anisomycin infused into the hippocampus fails to block "reconsolidation" but impairs extinction: The role of re-exposure duration. Learn. Mem. 13: 27-34.

Prusiner, S.B. 1998. Prions. Proc. Natl. Acad. Sci. 95: 13363-13383.

Przybyslawski, J., Roullet, P., and Sara, S.J. 1999. Attenuation of emotional and nonemotional memories after their reactivation: Role of $\beta$ adrenergic receptors. J. Neurosci. 19: 6623-6628.

Roberson, E.D. and Sweatt, J.D. 1999. A biochemical blueprint for long-term memory. Learn. Mem. 6: 381-388.

Rodriguez, W.A., Horne, C.A., and Padilla, J.L. 1999. Effects of glucose and fructose on recently reactivated and recently acquired memories. Prog. Neuropsychopharmacol. Biol. Psychiatry 23: $1285-1317$.

Roullet, P. and Sara, S. 1998. Consolidation of memory after its reactivation: Involvement of $\beta$-adrenergic receptors in the late phase. Neural Plast. 6: 63-68.

Saitoh, T. and Schwartz, J.H. 1985. Phosphorylation-dependent subcellular translocation of a $\mathrm{Ca}^{2+} /$ calmodulin-dependent protein kinase produces an autonomous enzyme in Aplysia neurons. J. Cell Biol. 100: 835-842.

Shimizu, E., Tang, Y.P., Rampon, C., and Tsien, J.Z. 2000. NMDA receptor-dependent synaptic reinforcement as a crucial process for memory consolidation. Science 290: 1170-1174.

Si, K., Giustetto, M., Etkin, A., Hsu, R., Janisiewicz, A.M., Miniaci, M.C., Kim, J.H., Zhu, H., and Kandel, E.R. 2003a. A neuronal isoform of
CPEB regulates local protein synthesis and stabilizes synapse-specific long-term facilitation in Aplysia. Cell 115: 893-904.

Si, K., Lindquist, S., and Kandel, E.R. 2003b. A neuronal isoform of the Aplysia CPEB has prion-like properties. Cell 115: 879-891.

Snyder, G.L., Allen, P.B., Fienberg, A.A., Valle, C.G., Huganir, R.L., Nairn, A.C., and Greengard, P. 2000. Regulation of phosphorylation of the GluR1 AMPA receptor in the neostriatum by dopamine and psychostimulants in vivo. J. Neurosci. 20: 4480-4488.

Steele, R.J. and Morris, R.G. 1999. Delay-dependent impairment of a matching-to-place task with chronic and intrahippocampal infusion of the NMDA-antagonist D-AP5. Hippocampus 9: 118-136.

Strekalova, T., Zorner, B., Zacher, C., Sadovska, G., Herdegen, T., and Gass, P. 2003. Memory retrieval after contextual fear conditioning induces c-Fos and JunB expression in CA1 hippocampus. Genes Brain Behav. 2: 3-10.

Summers, M.J., Crowe, S.F., and Ng, K.T. 1997. Administration of DL-2-amino-5 phosphonovaleric acid (AP5) induces transient inhibition of reminder-activated memory retrieval in day-old chicks. Brain Res. Cogn. Brain Res. 5: 311-321.

Sweatt, J.D. 2003. The chemistry of perpetual memory. In Mechanisms of memory, pp. 372-388. Elsevier Academic Press, New York. . 2004. Mitogen activated protein kinases in synaptic plasticity and memory. Curr. Opin. Neurobiol. 14: 311-317.

Taubenfeld, S.M., Milekic, M.H., Monti, B., and Alberini, C.M. 2001. The consolidation of new but not reactivated memory requires hippocampal C/EBPß. Nat. Neurosci. 4: 813-818.

Torras-Garcia, M., Lelong, J., Tronel, S., and Sara, S.J. 2005. Reconsolidation after remembering an odor-reward association requires NMDA receptors. Learn. Mem. 12: 18-22.

Tronson, N.C., Wiseman, S.L., Olausson, P., and Taylor, J.R. 2006. Bidirectional behavioral plasticity of memory reconsolidation depends on amygdalar protein kinase A. Nat. Neurosci. 9: 167-169.

Turner, B.M. 2002. Cellular memory and the histone code. Cell 111: $285-291$.

Valjent, E., Corbille, A.G., Bertran-Gonzalez, J., Herve, D., and Girault, J.A. 2006. Inhibition of ERK pathway or protein synthesis during reexposure to drugs of abuse erases previously learned place preference. Proc. Natl. Acad. Sci. 103: 2932-2937

von Hertzen, L.S. and Giese, K.P. 2005. Memory reconsolidation engages only a subset of immediate-early genes induced during consolidation. J. Neurosci. 25: 1935-1942.

Weiss, F., Maldonado-Vlaar, C.S., Parsons, L.H., Kerr, T.M., Smith, D.L. and Ben-Shahar, O. 2000. Control of cocaine-seeking behavior by drug-associated stimuli in rats: Effects on recovery of extinguished operant-responding and extracellular dopamine levels in amygdala and nucleus accumbens. Proc. Natl. Acad. Sci. 97: 4321-4326.

Wood, M.A., Kaplan, M.P., Park, A., Blanchard, E.J., Oliveira, A.M., Lombardi, T.L., and Abel, T. 2005. Transgenic mice expressing a truncated form of CREB-binding protein (CBP) exhibit deficits in hippocampal synaptic plasticity and memory storage. Learn. Mem. 12: 111-119.

Yeh, S.H., Lin, C.H., Lee, C.F., and Gean, P.W. 2002. A requirement of nuclear factor-кB activation in fear-potentiated startle. J. Biol. Chem. 277: 46720-46729.

Zhang, W.P., Guzowski, J.F., and Thomas, S.A. 2005. Mapping neuronal activation and the influence of adrenergic signaling during contextual memory retrieval. Learn. Mem. 12: 239-247. 


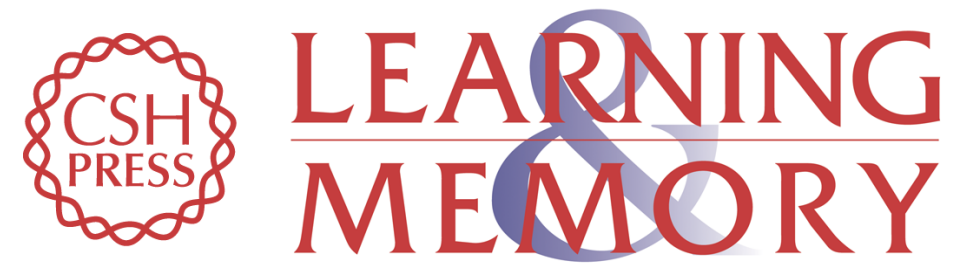

\section{Amnesia or retrieval deficit? Implications of a molecular approach to the question of reconsolidation}

Courtney A. Miller and J. David Sweatt

Learn. Mem. 2006, 13:

Access the most recent version at doi:10.1101//m.304606

References This article cites 75 articles, 31 of which can be accessed free at: http://learnmem.cshlp.org/content/13/5/498.full.html\#ref-list-1

License

Email Alerting Receive free email alerts when new articles cite this article - sign up in the box at the Service top right corner of the article or click here. 